\title{
"How Compliant Do We Want To Be With The Colonoscopy Surveillance Guidelines?"
}

\author{
KC Chivers, \\ Clincial Researcher \\ Willaim Harvey Hospital, East-Kent Hospital Trust, Kennington Rd, Willesborough, \\ Ashford, Kent. TN24 0LZ \\ PS Basnyat, \\ Consultant Colorectal Sugeon \\ Willaim Harvey Hospital, East-Kent Hospital Trust, Kennington Rd, Willesborough, \\ Ashford, Kent. TN24 0LZ \\ Corresponding Autor: PRADEEP.BASNYAT@ekht.nhs.uk \\ $\mathrm{N}$ Taffinder \\ Consultant Colorectal Sugeon \\ Willaim Harvey Hospital, East-Kent Hospital Trust, Kennington Rd, Willesborough, \\ Ashford, Kent. TN24 0LZ \\ Word Count: 1098
}

Key Words: Colorectal Cancer; Colonoscopy, Surveillance, Guidelines, BSG, ACPGBI 


\begin{abstract}
Objective: To assess the referral practice for surveillance colonoscopy amongst clinicians and to measure whether practice was inline with the current Association of Coloproctology of Great Britain and Ireland (ACPGBI) and the British Society of Gastroenterology (BSG) guidelines. Methods: A questionnaire was sent to members of the ACPGBI. Results: A total of 195 (49\%) clinicians responded, providing information on their referral habits with comments on where they deviated from the guidelines. Conclusions: The BSG and ACPGBI guidelines are well established amongst clinicians and generally accepted as best practice however the majority of clinicians deviate from the guidelines for particular clinical scenarios. In fact only $18 \%$ of respondents followed all recommendations for surveillance colonoscopy for patients with polyps, previous cancers and a family history.
\end{abstract}




\section{Introduction}

In 2002, the Association of Coloproctology of Great Britain and Ireland (ACPGBI) and the British Society of Gastroenterology (BSG) released a comprehensive list of recommendations for surveillance colonoscopy of high-risk groups ${ }^{1}$. The aim of this study was to assess the referral practice for surveillance colonoscopy amongst clinicians and measure whether practice was inline with the current guidelines.

\section{Materials and Methods}

In 2006, a questionnaire (see Appendix) was sent to 400 members of the Association of Coloproctology of Great Britain and Ireland via a posted-circular. In addition to information on their referral habits, the clinicians were asked to give comments and/or define where they deviated from the guidelines.

\section{Results}

A total of $195(49 \%)$ clinicians responded. The majority of responders were consultants $(193,99 \%)$ and from surgical specialities $(180,92 \%) .96 \%$ (188) were practising colonoscopists. Very few respondents agreed with all the guidelines, but colonoscopists varied in which guidelines they chose to follow.

Polyp Follow Up: Of the responders, 179 (92\%) followed the guidelines for surveillance after high-risk polyps. With $184(94 \%)$ and $166(85 \%)$ following the guidelines for medium- and low- risk polyps respectively (Figure 1). The exceptions and comments provided by respondents associated with adenomatous polyps are summarized in table 1 .

Cancer Follow Up: Some 144 (74\%) respondents screened patients with a previous colorectal cancer (CRC) five yearly as recommended in the guidelines, with $51(26 \%)$ stating they scoped more frequently. Only $64(32 \%)$ ceased surveillance at the age of 70 years. Of those who said they would scope patients past the age of 70: - 33 would scope to 75 years; 28 to 80 years; 2 to 85 years; and 44 until the patient was "too frail" (Figure 2).

Family History Follow Up: 176 (90\%) were happy to start performing colonoscopy on patients with at least one first degree relative, under the age of 45 years, at the age of 35-40 years however only $108(55 \%)$ would wait to re-scope at the age of 55 . Likewise, 169 (87\%) would begin scoping those patients with at least two FDR, of any age, at 35-40 years but only $114(58 \%)$ would wait until the age of 55 years for the second surveillance colonoscopy. Almost half of respondents felt it more appropriate to scope patients with a family history of colorectal cancer every five years. Many respondents would seek the advice of their local genetic services.

Only $18 \%$ of respondents followed all the ACPGBI and BSG recommendations for surveillance colonoscopy for patients with polyps, previous cancers and a family history. 


\section{Discussion}

Colonoscopy is the current gold standard for examination of colonic surfaces ${ }^{2}$. It is well established that regular colonoscopic surveillance of the bowel may allow early detection of CRC in high-risk groups ${ }^{3-8}$, even though one study captured $80 \%$ non compliance shortly after publication of the national guidelines ${ }^{9}$. The USA National Polyp Study observed the incidence of CRC in patients undergoing colonoscopic surveillance was $70-90 \%$ lower than expected compared with three reference populations ${ }^{10}$. Four years after the ACPGBI and BSG released their guidelines for colonoscopic surveillance ${ }^{1}$ almost all respondents said their normal practice was to follow some of the BSG/ ACPGBI guidelines when referring patients for surveillance colonoscopy, with all respondents being aware of the guidelines.

As far as we are aware this is the only study that examines the referral habits of colonoscopists across the country since the publication of the guidelines ${ }^{1}$. The overall response rate was reasonably high which we attribute the support offered by the ACPGBI. The main limitation of the study is the lack of data on the non-responders. As this was an anonymous questionnaire survey further information from the clinicians is not possible. We also contacted the British Society of Gastroenterology who sent the questionnaire to 2000 members of the via the delegate packs at the Society's annual meeting in Birmingham. We only received 13 responses $(0.65 \%)$ therefore the data is not included in this study. Questionnaires in delegate packs at large international conference is not a successful data collection method.

Overall, fewer respondents agreed with following up low-risk sigmoid adenomas compared to the recommendations for the high- and medium- risk adenomas. The majority of those who said they do not scope five yearly but preferred to scope more regularly.

Two thirds of all clinicians did not want to cease surveillance of cancer or polyp patients at the specified age. The cut off age is set at 70 years for patients with a previous cancer and 75 years for those patients with polyps as the remaining life expectancy is likely to be less than the average time required for new adenoma to become malignant ${ }^{2}$. With our aging population in Britain, clinicians reluctance to limit surveillance by age may be justified.

Two respondents commented on concerns of the reliability of patients being able to give a full, accurate family history of colorectal cancer. Aitken et al (1995) ${ }^{11}$ conducted a study to validate the self-reported family histories of colorectal cancer of 237 patients undergoing colonoscopy. They confirmed positive family histories in $77 \%$ of cases and estimated $98 \%$ of the negative family histories were correct $^{11}$.

Guidelines are seen by many as simply recommendations for practice by central organisations with the final decisions remaining with the treating clinician. High level of acceptance of these guidelines, for duration and frequency of surveillance colonoscopy, does hold the promise national acceptance thereby preventing postcode 
treatment. Three consultants referred to local guidelines within their trust and one reported he followed the American Societie's guidelines. These findings raise some interesting questions regarding practise outside recommendations of central organisations and informed consent of patients. With a perforation rate of $0.2 \%{ }^{12}$ as well as other risks including bleeding, infection etc; in the event of a complication, an informed lawyer would find it easy to attack a clinician operating outside wellestablished national guidelines. Interestingly, several practitioners stated lack of capacity and/or daunting waiting lists as factors preventing them performing colonoscopies at frequencies the would like or within the guidelines' recommendations.

To conclude, although the BSG and ACPGBI guidelines are well established amongst clinicians and generally accepted as best practice. However less than $1 / 5^{\text {th }}$ of the clinicians who responded follow all the guidelines. Perhaps the decision to perform each colonoscopy must also take into account the wishes of the individual patient, the presence of co-morbidity, local guidelines and other risk factors associated with the procedure. We believe that education of the guidelines among clinicians and nurses in the endoscopy department may improve uptake of guidelines and improve patient services.

\section{Acknowledgments}

We are grateful to the Association of Coloproctology of Great Britain and Ireland for their support in sending out our questionnaire. We also thank all the respondents for their help. 


\section{References}

1. Caines S, Scholefield JH. Guidelines for Colorectal Cancer Screening in High Risk Groups (Edited). GUT Supplement 2002;0(Suppl V):v1-v2.

2. Atkin WS, Saunders BP. Surveillance guidelines after removal of colorectal adenmatous polyps. Gut 2002;51(suppl V):v6-v9.

3. Khoury DA, Opelka FG, Beck DE, Hicks TC, Timmcke AE, Gathright JB, Jr. Colon surveillance after colorectal cancer surgery. Dis Colon Rectum 1996;39(3):252-6.

4. Zauber AG, Winawer SJ. Initial management and follow-up surveillance of patients with colorectal adenomas. Gastroenterol Clin North Am 1997;26(1):85-101.

5. Blumberg D, Opelka FG, Hicks TC, Timmcke AE, Beck DE. Significance of a normal surveillance colonoscopy in patients with a history of adenomatous polyps. Dis Colon Rectum 2000;43(8):1084-91; discussion 1091-2.

6. Eaden J, Abrams K, Ekbom A, Jackson E, Mayberry J. Colorectal cancer prevention in ulcerative colitis: a case-control study. Aliment Pharmacol Ther 2000;14(2):145-53.

7. Thiis-Evensen E, Hoff GS, Sauar J, Langmark F, Majak BM, Vatn MH. Population-based surveillance by colonoscopy: effect on the incidence of colorectal cancer. Telemark Polyp Study I. Scand J Gastroenterol 1999;34(4):414-20.

8. Belchetz LA, Berk T, Bapat BV, Cohen Z, Gallinger S. Changing causes of mortality in patients with familial adenomatous polyposis. Dis Colon Rectum 1996;39(4):384-7.

9. Shoaib A, Hamade A, Zia A, Basnyat PS, Taffinder N. Why Wait For A Colonoscopy? An Easy Cure. Colorectal Disease 2006;8(6):480-3.

10. Winawer SJ, Zauber AG, Ho MN, O'Brien MJ, Gottlieb LS, Sternberg SS, et al. Prevention of colorectal cancer by colonoscopic polypectomy. The National Polyp Study Workgroup. N Engl J Med 1993;329(27):1977-81.

11. Aitken J, Bain C, Ward M, Siskind V, MacLennan R. How accurate is selfreported family history of colorectal cancer? Am J Epidemiol 1995;141(9):863-71.

12. Anderson ML, Pasha TM, Leighton JA. Endoscopic perforation of the colon: lessons from a 10-year study. Am J Gastroenterol 2000;95(12):3418-22.

13. Single flexible sigmoidoscopy screening to prevent CRC: baseline findings of a UK multicentre randomised trial . Lancet 2002;359(9314):1291-30. 